\title{
Restabelecimento estético através de clareamento externo e faceta direta
}

\author{
Aesthetic restoration through external whitening and direct veener
}

Restauración estética através de blanqueamiento externo y chapa directa

\begin{abstract}
Vinícius Augusto Carneiro Pereira1 ${ }^{1}$, Sandson Cleyton Ferreira da Silva Oliveira², Rauhan Gomes de Queiroz ${ }^{3}$, Katryne Targino Rodrigues ${ }^{1}$, José Henrique de Araújo Cruz ${ }^{1 *}$, Débora Lorrany Rocha de Carvalho' ${ }^{1}$, Isadora da Costa Filgueira ${ }^{1}$, Elizandra Silva da Penha1 ${ }^{1}$, Luanna Abílio Diniz Melquíades de Medeiros¹, Gymenna Maria Tenório Guênes¹.
\end{abstract}

\section{RESUMO}

Objetivo: Apresentar o relato de um caso clínico, no qual se realizou o restabelecimento estético do elemento dentário 11, tratado endodonticamente, através do uso do clareamento externo associado à realização de uma faceta direta com resina composta. Relato do caso: Paciente do sexo masculino, 23 anos de idade, procurou tratamento estético queixando-se do escurecimento do elemento dentário 11 tratado endodonticamente. Foi realizado exame clínico comprovando alteração de cor e exame radiográfico com excesso de material obturador dentro da câmara pulpar. O plano de tratamento foi proposto para restabelecer função e estética do dente comprometido. Para tal finalidade, realizou-se rebaixamento de guta-percha, clareamento dental externo de consultório entre pré-molares de ambos os arcos dentários com concentração de $35 \%$ (FGM, Whiteness HP, Brasil) e realização de faceta direta em resina composta do elemento 11. Considerações finais: As etapas do tratamento revelaram que o planejamento correto combinado com o conhecimento das técnicas disponíveis e das propriedades dos materiais é de significativa importância para a obtenção de um resultado estético satisfatório, possibilitando a reabilitação do elemento dental.

Palavras-chave: Clareamento dental, Dente não vital, Estética dentária.

\begin{abstract}
Objective: to present a report of a clinical case in which the aesthetic restoration of the endodontically treated dental element 11 was performed through the use of external bleaching associated with a direct facet of composite resin. Case report: A 23-year-old male patient sought care for aesthetic treatment complaining about the darkening of the endodontically treated dental element. A clinical and radiographic examination was performed, proving color change and identification of excess obturator material inside the root canal. The treatment plan was proposed to restore function and esthetics of the involved tooth. For this purpose, a lowering of gutta-percha, external dental bleaching between premolars of both dental arches with a concentration of 35\% (FGM, Whiteness HP, Brazil) and a direct facet of composite resin of element 11 were performed. Final considerations: Treatment stages revealed that the correct planning combined with the knowledge of available techniques and material properties is of significant importance in order to obtain a satisfactory aesthetic result, making possible the rehabilitation of the dental element.
\end{abstract}

Key words: Dental whitening, Non-vital tooth, Dental esthetics.

1Universidade Federal de Campina Grande (UFCG), Patos - PB. *E-mail:henrique_araujo1992@hotmail.com 2Universidade Federal da Paraíba (UFPB), João Pessoa - PB.

${ }^{3}$ Universidade Federal do Ceará (UFC), Fortaleza - CE. 


\section{RESUMEN}

Objetivo: Presentar el relato de un caso clínico, en el cual se realizó el restablecimiento estético del elemento dental 11, tratado endodónticamente, a través del uso del blanqueamiento externo asociado a la realización de una faceta directa con resina compuesta. Relato del caso: Paciente del sexo masculino, 23 años, buscó atención para tratamiento estético quejándose del oscurecimiento del elemento dental 11 endodónticamente tratado. Se realizó un examen clínico y radiográfico, comprobando alteración de color e identificación de exceso de material obturador dentro del conducto radicular. El plan de tratamiento fue propuesto para restablecer la función y la estética del diente comprometido. Para tal fin, se realizaron descenso de gutapercha, blanqueamiento dental externo entre premolares de ambos arcos dentales con concentración de 35\% (FGM, Whiteness HP, Brasil) y realización de faceta directa en resina compuesta del elemento 11. Consideraciones finales: Las etapas del tratamiento revelaron que la planificación correcta combinada con el conocimiento de las técnicas disponibles y de las propiedades de los materiales es de significativa importancia para la obtención de un resultado estético satisfactorio, posibilitando la rehabilitación del elemento dental.

Palabras clave: Blanqueamiento dental, Diente no vital, Estética dental.

\section{INTRODUÇÃO}

A progressiva demanda por tratamentos restauradores estéticos aliados aos recentes avanços na Odontologia adesiva, levaram a criação de materiais e técnicas, buscando restaurar a aparência natural do dente, especialmente na região dos elementos anteriores (MIGLIAU G, et al., 2015).

Desde que as técnicas adesivas foram incorporadas à Odontologia, os procedimentos restauradores tornaram-se mais conservadores. No passado, as alternativas mais viáveis na solução do problema de escurecimento dental eram basicamente a aplicação de diferentes técnicas protéticas. Além disso, com o proporcionamento de resultados estéticos questionáveis, na maioria das vezes, era necessária a remoção excessiva de estrutura dentária sadia (SANTOS RL, et al., 2010).

Contudo, com o advento da nova filosofia da Odontologia Restauradora, preconiza-se que, para qualquer tipo de procedimento, o cirurgião-dentista deve sempre optar pelo tratamento mais conservador, ou seja, aquele que proporcione maior preservação de estrutura dentária saudável (CALABRIA MP, et al., 2011).

Atualmente, há diversas opções para reabilitar a estética do sorriso. Entre elas, encontram-se técnicas menos invasivas, como o clareamento dental, até restaurações indiretas, as quais implicam desgaste maior dos elementos dentários. Entretanto, para que se obtenha um bom tratamento estético, é necessário que o profissional detenha conhecimentos sobre estética, materiais restauradores e técnicas disponíveis (WENDLING M, et al., 2016).

Para satisfazer as necessidades estéticas no tratamento restaurador, nos deparamos com a possibilidade do uso das resinas compostas. Portanto, diversos requisitos devem ser observados a fim de garantir a longevidade da restauração e o sucesso clínico do tratamento restaurador adesivo direto (SILVA GR, et al., 2018).

Então, diante de um elemento dentário tratado endodonticamente que apresente qualquer tipo de escurecimento dental, o cirurgião-dentista pode utilizar alguns tipos de terapias conservadoras, como o clareamento dental e a reanatomização dentária para recuperar a estética do dente através de técnicas restauradoras, ou, ainda, unir a técnica restauradora com a do clareamento (LUCENA MTL, et al., 2018).

Sendo assim, este trabalho tem como objetivo apresentar o relato de um caso clínico, no qual se realizou o restabelecimento estético do elemento dentário 11, tratado endodonticamente, através do uso do clareamento externo associado à realização de uma faceta direta com resina composta.

\section{RELATO DE CASO}

Paciente, gênero masculino, 23 anos de idade, procurou a clínica escola de Odontologia da Universidade Federal de Campina Grande (UFCG) queixando-se do escurecimento do incisivo central superior direito. 
Durante a anamnese, o paciente relatou trauma do referido elemento após queda, sofrida na infância, com consequente realização de tratamento endodôntico.

Ao exame clínico, pode-se observar uma nítida alteração de cor do dente em questão quando comparado aos elementos dentários adjacentes. Em consonância, com auxílio do exame radiográfico, notou-se a presença de tratamento endodôntico satisfatório, mas com considerável excesso de material obturador no interior da câmara pulpar, estendendo-se até aproximadamente um terço da câmara coronária. Além disso, observou-se também que os demais dentes apresentavam coloração amarelada em diferentes tons (Figura 1A).

Sendo assim, o plano de tratamento foi traçado com a finalidade de estabelecer uma estética mais satisfatória para a paciente, bem como proporcionar resistência ao elemento 11. Inicialmente, foi realizada abertura coronária com ponta esférica diamantada № 1012 (KG Sorensen ${ }^{\circledR}$, São Paulo, Brasil) do referido dente e a limpeza de todo o remanescente do teto da câmara pulpar. Em seguida, realizou-se o rebaixamento de Guta-percha com auxílio dos calcadores de Paiva (Millennium - Golgran $\AA$, Brasil) aquecidos em lamparina (Figura 1B). Uma vez que o excesso de material obturador foi removido suficientemente, uma nova tomada radiográfica foi realizada para simples conferência (Figura 1C).

Figura 1 - Radiografia periapical inicial (A), Visão da câmara pulpar (B), Radiografia periapical final (C).

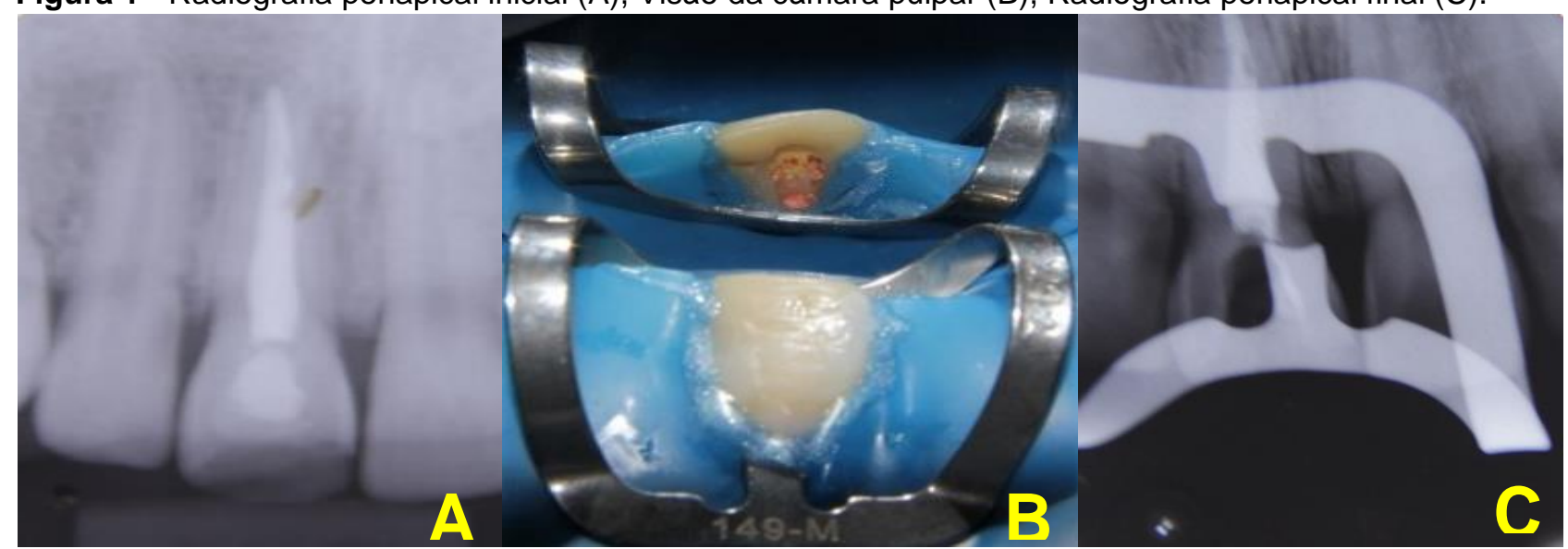

Fonte: Pereira VAC, et al., 2020.

Em seguida, procedeu-se com a limpeza interna da câmara pulpar com a aplicação de ácido fosfórico a $37 \%$ (Allplan ${ }^{\circledR}$, Brasil) por 15 segundos e lavagem com água destilada pelo dobro de tempo. Depois, foram aplicadas duas camadas do adesivo Single Bond 2 (3M/ESPE ${ }^{\circledR}$, São Paulo, Brasil), sob fotopolimerização na segunda camada por 20 segundos e confecção da restauração permanente com resina composta na cor A2 Z350 (3M/ESPE ${ }^{\circledR}$, São Paulo, Brasil).

Após a remoção do isolamento absoluto, foram verificados os contatos oclusais com a finalidade de se evitarem interferências que pudessem ser responsáveis por uma sobrecarga na restauração definitiva, determinando a sua falha ou fratura coronária posteriormente. De forma imediata, após a efetivação de todo o protocolo já realizado, pode-se observar uma nítida melhora da tonalidade do elemento 11 (Figura 2A).

Sucedendo-se essa etapa do planejamento do tratamento estético, foi realizada a seleção de cor dos dentes sob auxílio de uma escala de cores (Vitapan ${ }^{\circledR}$ Classical) e do registro fotográfico que, por sua vez, são ferramentas importantes no auxílio da documentação do tratamento e acompanhamento dos resultados.

Para o clareamento externo de consultório dos dentes, foi utilizado um gel à base de peróxido de hidrogênio a 35\% (FGM, Whiteness HP, Brasil). A fim de evitar o contato do gel clareador com os tecidos gengivais, foi utilizada a barreira gengival top dam (FGM, Whiteness HP, Brasil), a qual, após adaptação por toda a margem gengival foi polimerizada. Em seguida, também se aplicou duas camadas de dessensibilizante (Biodinâmica ${ }^{\circledR}$, São Paulo, Brasil), a fim de diminuir a sensibilidade causada pelo agente clareador. Após proteção gengival e isolamento relativo, nessa primeira sessão, todas as superfícies 
vestibulares dos dentes, de segundo pré-molar a segundo pré-molar (superior e inferior) foram condicionados com o agente clareador conforme as recomendações determinadas pelo fabricante. $\mathrm{O}$ mesmo processo se repetiu após 7 e 14 dias, respectivamente (Figura 2B-2C).

Figura 2 - Seleção de cor inicial (A), clareamento externo da arcada superior (B), clareamento externo da

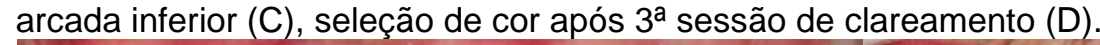

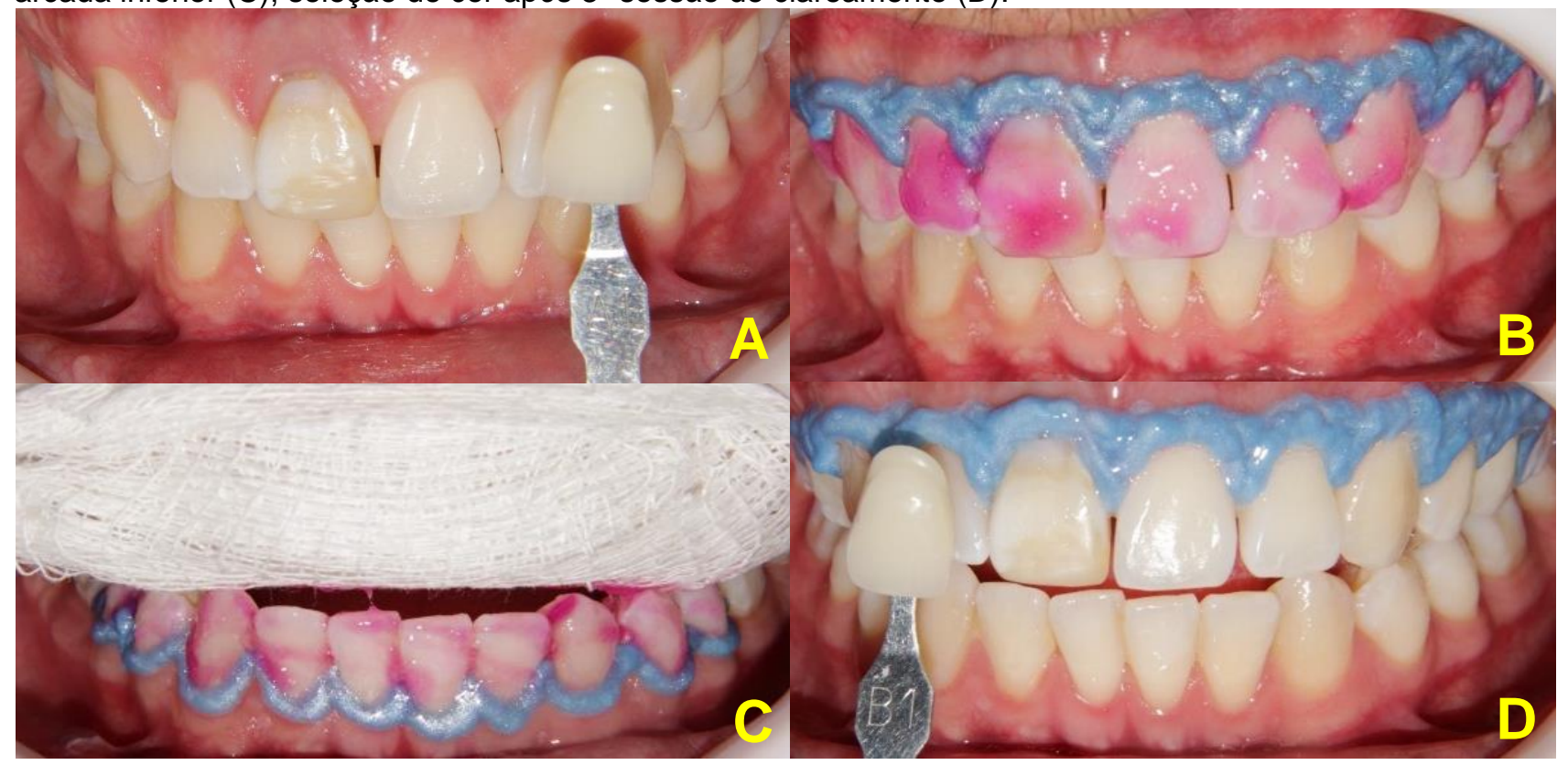

Fonte: Pereira VAC, et al., 2020.

Após 14 dias da última aplicação do gel clareador, para estabilização da cor dos elementos dentários, foi feita o registro de cor através da escala Vita (Vitapan ${ }^{\circledR}$ Classical), no qual o elemento se apresentava na cor B1 (Figura 2D). Então foi inserido o fio retrator \#00 (Biodinâmica ${ }^{\circledR}$, São Paulo, Brasil) no elemento 11 (Figura 3A), e com pontas diamantadas № 1012, № 2135 e № 2135F (KG Sorensen ${ }^{\circledR}$, São Paulo, Brasil) foi feito desgaste a nível de esmalte, para promover retenção à restauração. Seguiu-se com a aplicação do ácido fosfórico a 37\% (Allplan ${ }^{\circledR}$, Brasil) por 30 segundos (Figura 3B) e lavagem com água destilada pelo dobro de tempo. Em seguida, foram feitas aplicações de duas camadas do adesivo Single Bond 2 (3M/ESPE ${ }^{\circledR}$, São Paulo, Brasil), sob fotopolimerização na segunda camada por 20 segundos (Figura 3C).

Figura 3 - Preparo do elemento dentário com inserção de fio retractor (A), condicionamento ácido (B), Sistema adesivo $(\mathrm{C})$.

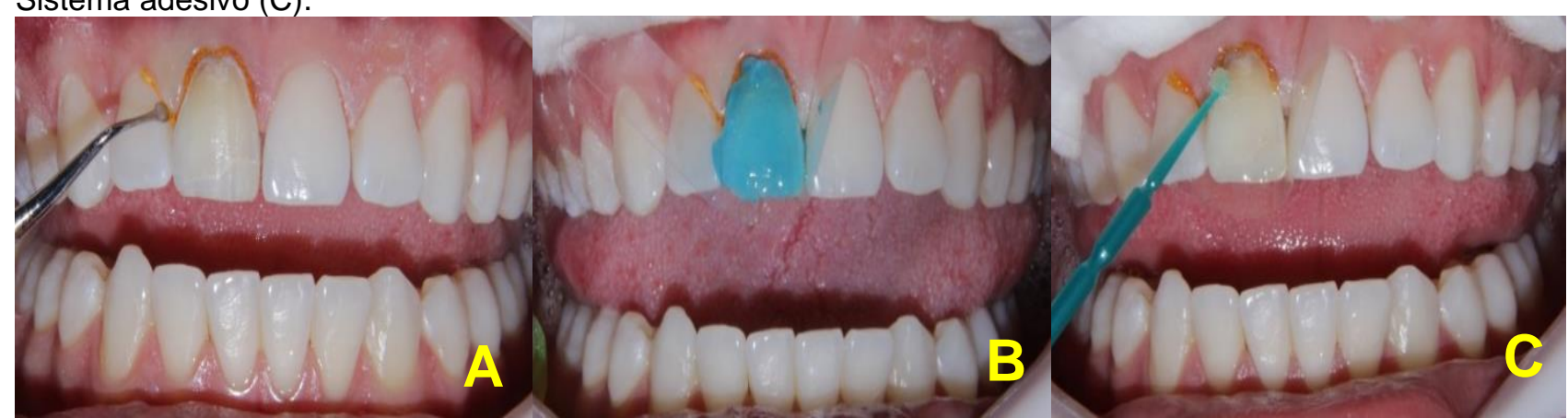

Fonte: Pereira VAC, et al., 2020.

Com o elemento dentário pronto para receber a resina composta, foram inseridos pequenos incrementos da resina de corpo na cor B1 Z350 (3M/ESPE ${ }^{\circledR}$, São Paulo, Brasil) e fotopolimerizando a cada inserção do material restaurador. Na última camada, foi utilizado a resina de esmalte na cor A1 Z350 (3M/ESPE ${ }^{\circledR}$, São Paulo, Brasil). Ao fim da inserção dos incrementos, foi feita uma última fotopolimerização. 
Figura 4: Aspecto final do sorriso após polimento da restauração.

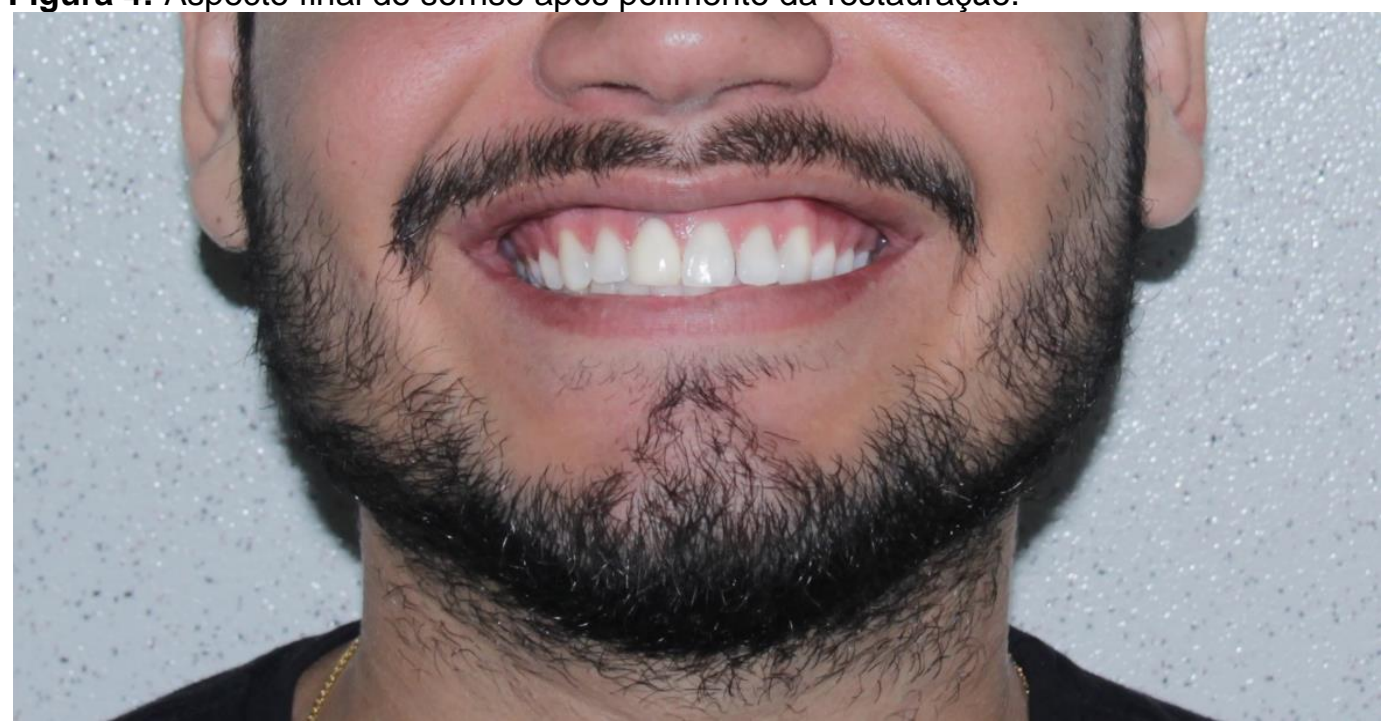

Fonte: Pereira VAC, et al., 2020.

Depois de 48 horas, o paciente retornou à clínica-escola para acabamento e polimento da restauração. Utilizou-se, inicialmente, a ponta diamantada № 3138F (KG Sorensen ${ }^{\circledR}$, São Paulo, Brasil), para remoção de excessos na região cervical do dente e com 3 discos de granulações diferentes (TDV ${ }^{\circledR}$, São Paulo, SP, Brasil) foi realizado o refinamento da restauração. O polimento foi feito com taças (American Burrs $^{\circledR}$, Brasil) em 3 granulações diferentes e disco de feltro (TDV ${ }^{\circledR}$, São Paulo, SP, Brasil) com pasta Diamond R (KG Sorensen ${ }^{\circledR}$, São Paulo, Brasil) dando brilho e um aspecto mais natural ao elemento dentário (Figura 4).

\section{DISCUSSÃO}

A maioria dos procedimentos estéticos na Odontologia têm por finalidade, devolver forma e função às estruturas bucais, com o objetivo de proporcionar conforto emocional ao paciente através da harmonização do sorriso (REZENDE MCRA e FAJARDO RS, 2016; GOMES GLS, et al., 2017.)

A forma do dente, a cor e a textura da superfície são critérios estéticos fundamentais a serem considerados no design do sorriso. Além disso, atualmente, a revolução das técnicas conservadoras da odontologia estética para manter a estrutura dentária o mais natural possível, tornou o tratamento mais confortável e adequado para os pacientes (ALBARRAK AA, et al. 2019). No presente estudo, o paciente relatou desconforto estético quanto a coloração e forma do elemento 11. Nessa perspectiva, foi preconizado um plano de tratamento respeitando seu desejo de um dente mais harmônico esteticamente.

Embora a literatura ainda se apresente escassa, Mendes BMS, Albino LGB e Rodrigues JA (2011) apontam que, o rebaixamento de gutta-percha possui finalidade de diminuir o escurecimento do remanescente dentário. Essa medida é preconizada, pelo fato de se mostrar como uma resposta mais rápida quando comparada à ausência de tal conduta.

Assim, como citado pelos referidos autores, essa tomada de decisão corrobora com o caso clínico aqui apresentado, uma vez que foi realizado o rebaixamento de gutta-percha antes do clareamento dental com a finalidade de remoção do excesso desse tipo de material endodôntico, que estava causando o escurecimento do dente.

Embora o agente clareador tenha capacidade de remover pigmentos orgânicos, sua resposta costuma ser mais demorada caso realizado primeiro ou, caso contrário, haveria a possibilidade de recidiva e o agente clareador poderia não conseguir remover toda a pigmentação.

Após remoção do agente responsável pela alteração de cor do elemento 11, foi proposto um clareamento externo para uniformizar as cores dos elementos dentários do paciente. Semelhante ao que 
este caso clínico propôs como plano de tratamento, Migliau G, et al. (2015) relataram o caso clínico de um incisivo central escurecido também associado a trauma na infância, cujo tratamento endodôntico estava insatisfatório. Diferentemente do que foi proposto aqui, os autores realizaram o retratamento do canal radicular, com posterior clareamento interno e restauração coronária.

$\mathrm{Na}$ técnica de clareamento dental interno em dentes não vitais, pode ocorrer um extravasamento do material clareador, promovendo um processo inflamatório na junção amelocementária. Esses agentes podem estimular o desenvolvimento de uma reabsorção cervical externa, em decorrência da exposição dos túbulos dentinários cervicais, modificando bioquimicamente os tecidos dentários de suporte dessa região. (MACIEL KBL, BARBOSA JS E LINS FF, 2018; MILESKY T, et al., 2018; CANUTO LC, et al., 2020).

Porém, já se sabe que reabsorção radicular externa é um efeito adverso que pode ser desencadeada pelo clareamento dental interno e/ou trauma. O clareamento dental externo é um método que também pode ser empregado em dentes não vitais. Quando comparado a segurança da técnica, observa-se que o risco de efeitos adversos é muito menor na técnica externa (MONTEIRO RV, JUNIOR SM e ANDRADA MAC, 2018; ROKAYA ME, et al., 2015). Corroborando com o estudo feito por Mendes BMS, Albino LGB e Rodrigues JA (2011), justificando a escolha em fazer apenas o clareamento externo e diminuindo os riscos de futuros problemas de reabsorção radicular externa.

Segundo Espíndola-Castro LF, et al. (2018) o peróxido de hidrogênio de $20 \%$ a $38 \%$ é o produto mais indicado para clareamento externo realizada em consultório. O protocolo são três aplicações do agente clareador, substituindo o gel a cada 15 minutos. Para o presente estudo, foi utilizado o peróxido de hidrogênio à $35 \%$ com três aplicações do gel clareador, corroborando também com o estudo feito por Neto JMA, et al. (2020) que diz que é responsabilidade do cirurgião-dentista a escolha correta do agente clareador, assegurando não só a parte harmônica do sorriso, como também a sua função.

A espessura da dentina e do esmalte tendem a variar de acordo com os diferentes grupos de dentes e também das diferentes partes da coroa, no qual a aparência natural dos dentes depende de sua espessura, calcificação, composição e translucidez. Essas características são responsáveis pelas tonalidades policromáticas do elemento dentário (TURGUT S, et al., 2018).

Em decorrência disso, no presente caso, foram utilizados três tipos de resinas compostas: uma resina (cor universal) para opacificar o substrato dentário, uma resina de corpo (cor B1) e outra de esmalte (cor A1). A tomada de decisão pelas cores mencionadas foi realizada para que ocorresse a mimetização do elemento restaurado com os dentes adjacentes, tanto em tonalidade de cor como em simetria.

A técnica direta possui vantagens e entre elas, estão: o tempo clínico; a conservação dos tecidos dentais; possibilidade de reparo; menos agressão aos tecidos periodontais; menor custo; possibilidade de controlar a cor; e, a forma do dente restaurado (SILVA GR, et al., 2018). Nessa perspectiva, o presente caso corrobora com o estudo mencionado, uma vez que se optou por uma técnica mais viável para as condições de fragilidade do elemento dentário.

Com isso, a realização de procedimentos pré-operatórios, seleção do material, seleção de cor, isolamento eficaz, preparo dentário adequado, inserção correta dos incrementos resinosos, são indispensáveis para obter-se êxito no resultado final das restaurações diretas e um resultado mais próximo do natural possível (LIMA MGS, et al., 2019). No caso clínico supracitado, corroborou-se com o presente estudo sobre todas estas etapas para conseguir um resultado final satisfatório, atendendo as expectativas do paciente e devolvendo estética, forma e função ao elemento dentário.

$\mathrm{Na}$ Odontologia moderna, se convencionou técnicas menos invasivas capazes de aliar a estética com a preservação de tecidos sadios. Portanto, um correto planejamento a ser instituído, bem como domínio das técnicas e conhecimento dos materiais restauradores, é possível devolver uma estética mais harmoniosa ao sorriso, aumentando a autoestima do paciente. Sendo assim, são necessários mais trabalhos multidisciplinares entre dentística e endodontia, para que se obtenha um protocolo que seja mais conservador, de menor custo e que garanta uma estética favorável. 


\section{REFERÊNCIAS}

1. ALBARRAK AA, et al. Multidisciplinary approach with predictable esthetics: A case report. The Saudi dental journal. 2019; 31: 89-95.

2. CALABRIA MP, et al. Restabelecimento estético e funcional de incisivo central superior traumatizado por meio de clareamento dentário e cimentação de pino intracanal. Controle de 2 anos. Rev Dent Press de Estética. 2011; 8(3): 74-88.

3. CANUTO LC, et al. Clareamento dental interno: relato de caso. Revista Eletrônica Acervo Saúde. 2020; 48 : e3236.

4. ESPÍNDOLA-CASTRO LF, et al. Influência da mudança de protocolo de clareamento dentário em consultório no resultado estético: caso clínico. Revista UNINGÁ. 2018; 55(3): 130-139.

5. GOMES GLS, et al. Restaurações adesivas com resina composta: durabilidade da linha de união. Rev Odontol Univ São Paulo. 2017; 22(1): 56-64.

6. LIMA MGS, et al. Reanatomização do sorriso com uso de resina composta: relato de caso. Archives of Health Investigation. 2019; 08: 501-505.

7. LUCENA MTL, et al. Clareamento interno em dentes desvitalizados com a tecnica walking bleach - relato de caso. Rev Uni Review. 2018; 24(1): 33-39.

8. MACIEL KBL, et al. Clareamento em um dente desvitalizado: relato de caso. Revista Eletrônica Acervo Saúde. 2018; 18: e83.

9. MENDES BMS, et al. Clareamento externo de dente não vital. Revista Saúde-UNG-Ser. 2011; 5(2): 46-55.

10. MIGLIAU G, et al. Endo-restorative treatment of a severly discolored upper incisor: resolution of the "aesthetic" problem through Componeer veneering System. Ann Stomatol. 2015; 6(3-4): 113-8.

11. MILESKI T, et al. Clareamento interno em dente traumatizado: relato de caso clínico. Revista UNINGÁ. 2018; 55(2): 24-32.

12. MONTEIRO RV, et al. Técnica de clareamento caseiro modificada para dente não vital: relato de caso. Rev Saúde Ci On. 2018; 7(1): 86-93.

13. NETO, JMAS, et al. Clareamento dental, aplicação em dentes vitais: uma revisão de literature. Revista Eletrônica Acervo Saúde. 2020; 47: e3086.

14. REZENDE MCRA, FAJARDO RS. Abordagem estética na Odontologia. Arch Health Invest. 2016; 5(1): 50-55.

15. ROKAYA ME, et al. Evaluation of Extraradicular Diffusion of Hydrogen Peroxide during Intracoronal Bleaching Using Different Bleaching Agents. Int J Dent. 2015; 2015.

16. ROZADO JB, et al. Reabilitação oral com facetas diretas em paciente com manchamento dental por tetraciclina: relato de caso. Braz. J. of Develop. 2020; 6(1): 3836- 3853.

17. SANTOS RL, et al. Solução estética através da interação do clareamento endógeno e restauração em resina composta. Rev Bra de Ci da Saúde. 2010; 13(3): 99-104.

18. SILVA GR, et al. Tratamento estético com diretas de resina composta - relato de caso. Rev Uni Review. 2018; 24(3): 27-31.

19. TURGUT S, et al. Color relationships of natural anterior teeth: An in vivo study. Niger J Clin Pract. 2018; 21: 925-31.

20. WENDLING M, et al. Reabilitação Funcional e Estética em Dentes Anteriores e Posteriores Relato de Caso. Clín. int. j. braz. Dente. 2016; 12(2): 172-79. 